\title{
Promising Therapeutic Effects of Cell Sheet Implantation with a Pedicle Omentum Flap to Enhance the Angiogenic Response to Ischemic Cardiomyopathy
}

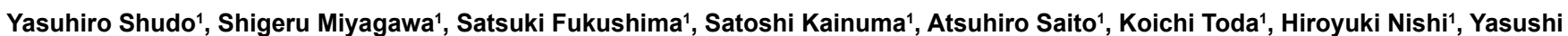
Yoshikawa ${ }^{1}$, Akima Harada ${ }^{1}$, Toshihiko Asanuma ${ }^{3}$, Satoshi Nakatani ${ }^{3}$, Tatsuya Shimizu², Teruo Okano ${ }^{2}$ and YoshikiSawa1*

${ }^{1}$ Department of Cardiovascular Surgery, Osaka University Graduate School of Medicine, Suita, Osaka, Japan

2Institute of Advanced Biomedical Engineering and Science, Tokyo Women's Medical University, Tokyo, Japan

3Department of Health Sciences, Division of Functional Diagnostics, Osaka University Graduate School of Medicine, Suita, Japan

\begin{abstract}
Introduction: We previously reported that skeletal myoblast (SMB) sheets induced the functional recovery of distressed myocardium in a large or small animal heart failure model via the paracrine effect of cytokines; however, this method was limited by poor vascular network and cell retention. We hypothesized that wrapping the cell sheet with pedicle omentum - a well-vascularized organ delivering different stem cell types and releasing various angiogenic and anti-inflammatory factors-might support retention of the implanted cells and enhance the therapeutic effects of the cell sheet technique on ischemic cardiomyopathy (ICM).

Methods and results: The ICM model was generated by inducing anterior myocardial infarction for 4 weeks in mini-pigs; whereas the scaffold-free cell sheets were generated from autologous SMBs in vitro. The cell sheets were placed on the infarct area with or without pedicle omentum wrapping. Mini-pigs that received the omentum flap only or no treatment were used as controls. The quantity of ironoxide-labeled transplanted cells was significantly greater with omentum wrapping than without at 8 weeks after treatment $(60 \pm 9 \%$ vs. $32 \pm 4 \%)$ as determined by magnetic resonance imaging. Implantation of the cell sheet wrapped with omentum significantly improved the left ventricle systolic function, increased blood perfusion, and accelerated therapeutic vessel growth into the host ischemic myocardium. The inflammatory effect was more attenuated following implantation of the cell sheet wrapped with omentum.
\end{abstract}

Conclusions: Use of the SMB cell sheet wrapped with pedicle omentum flap enhanced cell retention and promoted mature and functional microvasculature in porcine ICM model, thereby improving myocardial function.

Keywords: Heart failure; Angiogenesis; Cardiomyopathy

\section{Introduction}

Heart failure remains a frequent and life-threatening disorder despite recent medical and surgical advances. Myocardial regenerative therapy is attracting growing interest as a means to improve left ventricular (LV) function in patients with end-stage heart disease [13]. We previously reported that the use of myoblast sheets induced functional recovery of distressed myocardium in a large or small animal heart failure model via the paracrine effect of cytokines, but this method is limited by poor vascular network and cell retention [4]. A key point of improving cell retention may involve enhancing angiogenesis to protect against the ischemic injury of implanted cells and reduce inflammatory response after in vivo implantation. To this end, the omentum, a well-vascularized organ that delivers several kinds of stem cells and releases a variety of angiogenic and anti-inflammatory factors, can potentially provide revascularization and attenuate inflammation of the ischemic myocardium [5]. Accordingly, we speculated that the use of omentum might enhance implanted cell retention by improving angiogenesis and attenuating inflammation.

Although we recently described "the cell sheet covered with an omentum flap" technique as a new treatment strategy and reported that the combination of a myoblast sheet with omentum enhanced myocardial repair in a porcine infarction model, the detailed real-time phenomena and mechanistic insight have not been fully elucidated in vivo [6]. In the present study, to prove the mechanisms underlying combination therapy, we hypothesized that wrapping of skeletal myoblast (SMB) cell sheets with the pedicle omentum might support retention of the implanted cells by accelerating angiogenesis or antiinflammatory effects and enhancing the therapeutic effects of the cell sheet technique on ischemic cardiomyopathy (ICM).

\section{Materials and Methods}

Mini-pig ischemic cardiomyopathy models and study protocol

Twenty-four mini-pigs (female, 8-10 months old, 20-25 kg, Japan Farm Co. Ltd.) were used in this study. The animals were anesthetized with an intravenous administration of ketamine $(6 \mathrm{mg} / \mathrm{kg})$ and sodium pentobarbital $(10 \mathrm{mg} / \mathrm{kg})$ for endotracheal intubation and then maintained with inhaled sevoflurane (1-2\%). The pericardial space was exposed by left thoracotomy through the $4^{\text {th }}$ intercostal space. The distal portion of the left anterior descending coronary artery (LAD) was

*Corresponding author: Yoshiki Sawa, MD, PhD, Department of Cardiovascular Surgery, Osaka University Graduate School of Medicine, Osaka, Japan, Tel: +816-6879-3154; Fax: +81-6-6879-3163; E-mail: sawa-p@surg1.med.osaka-u.ac.jp

Received December 12, 2013; Accepted January 17, 2014; Published January 20, 2014

Citation: Shudo Y, Miyagawa S, Fukushima S, Kainuma S, Saito A, et al. (2014) Promising Therapeutic Effects of Cell Sheet Implantation with a Pedicle Omentum Flap to Enhance the Angiogenic Response to Ischemic Cardiomyopathy. J Stem Cell Res Ther 4: 159. doi:10.4172/2157-7633.1000159

Copyright: ( 2014 Shudo Y, et al. This is an open-access article distributed under the terms of the Creative Commons Attribution License, which permits unrestricted use, distribution, and reproduction in any medium, provided the original author and source are credited. 
Citation: Shudo Y, Miyagawa S, Fukushima S, Kainuma S, Saito A, et al. (2014) Promising Therapeutic Effects of Cell Sheet Implantation with a Pedicle Omentum Flap to Enhance the Angiogenic Response to Ischemic Cardiomyopathy. J Stem Cell Res Ther 4: 159. doi:10.4172/21577633.1000159

Page 2 of 7

directly ligated, followed by the placement of an ameroid constrictor around the LAD just distal of the left circumflex coronary artery branching to induce ICM [7].

Four weeks after ameroid constrictor placement, the mini-pigs were again given general anesthesia for echocardiography followed by treatment. Eight weeks after the treatment, the mini-pigs were again given general anesthesia for echocardiography, magnetic resonance imaging (MRI), angiography, and X-ray micro-computed tomography (micro-CT). The animals were then euthanized by intravenous injection of $200 \mathrm{mg} / \mathrm{kg}$ of pentobarbital and $2 \mathrm{mEq} / \mathrm{kg}$ of potassium chloride under terminal anesthesia, and the heart was excised (Figure $1)$.

\section{Preparation and grafting skeletal myoblast cell sheets}

Autologous skeletal muscle weighing approximately 10-15 g was removed from the quadriceps femoris muscle, and autologous SMB cells were cultured for 3 weeks to prepare for implantation as previously described [8]. SMB cells were incubated in $60-\mathrm{mm}$ temperatureresponsive culture dishes (UpCell ${ }^{\circledR}$; Cellseed, Tokyo, Japan) at $37^{\circ} \mathrm{C}$ and $5 \% \mathrm{CO}_{2}$ for 24 hours with the cell numbers adjusted to $1.5 \times 10^{7}$ cells per dish. The dishes were then transferred to another incubator set at $20^{\circ} \mathrm{C}$ for one hour to release the cultured cells as an intact cell sheet. Consequently, the SMB spontaneously detached to generate a free-floating monolayer cell sheet.

\section{Skeletal myoblast cell sheets with omentum implantation}

The mini-pigs with MI were divided into four groups ( $\mathrm{n}=6$ each): SMB cell sheets wrapped with omentum (Cell sheet+Omentum group), SMB cell sheets only (Cell sheet group), omentum only (Omentum group), and no treatment (ICM group). Each animal in the Cell sheet+Omentum and Cell sheet groups received 30 SMB cell sheets, a total of $4.5 \times 10^{8}$ cells.

In the Cell sheet group, a median sternotomy was performed to implant the cell sheets onto the heart. The cell sheets were simply placed on the epicardium of the ischemic area (LAD region). In the Cell sheet+Omentum group, a small upper midline laparotomy and median sternotomy were performed to move the omentum from the peritoneal space into the mediastinal space, and care was taken to preserve the arch structure of the left gastroepiploic artery (GEA). We created the hole at the diaphragm and passed the omentum flap through the hole into the pericardial cavity. The SMB cell sheets were wrapped and

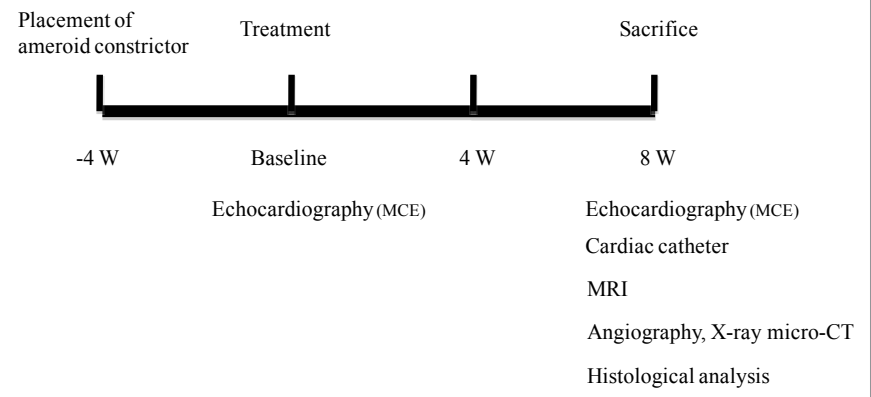

MCE: Myocardial Contrast Echocardiography; MRI: Magnetic Resonance Imaging

Figure 1: Experimental study design. Schematic outline of the timing of the cell culture along with the animal surgery, treatment, and outcome measurements. covered with the harvested omental flap, and a maximum of 5 SMB cell sheets could be implanted in a single location $[9,10]$. The procedure was conducted under general anesthesia.

\section{Echocardiography}

Blood perfusion analysis by performing myocardial contrast echocardiography: A myocardial contrast echocardiography (MCE) examination was performed by using Sonazoid (Daiichi-sankyo, Tokyo, Japan) as the contrast agent as described previously [11-13]. LV short axis images at the mid-LV level (clearly depicting the base of the papillary muscles) were obtained just before and 8 weeks after the treatment by using an ultrasonographic scanner (Aplio). MCE images were obtained in the intermittent mode every 4 cardiac cycles in which the end-systolic phase was triggered and were recorded as TIFF files. The video intensity (VI) of the anterior wall was analyzed by using Image Lab software (Toshiba). A round region of interest, $1 \mathrm{~cm}$ in diameter, was set at the anterior myocardium, the VI was measured in 3 consecutive end-systolic frames, and the average was calculated before and after contrast injection. The change in VI was calculated by subtracting the baseline intensity from the peak intensity on the MCE image.

\section{Hemodynamic studies by performing cardiac catheterization}

Pressure and volume measurements: Hemodynamic studies were performed to assess systolic and diastolic LV function 8 weeks after the treatment. The mini-pigs were anesthetized and placed in a supine position on a controlled heating pad, and then core temperature measured with a rectal probe was maintained at $37 \pm 0.5^{\circ} \mathrm{C}$. A MicroTip catheter transducer (SPR-671; Millar Instruments, Inc., Houston, TX, USA) and conductance catheters (Unique Medical Co., Tokyo, Japan) were placed longitudinally in the LV from the apex and connected to an Integral 3-signal conditioner-processor (Unique Medical Co.). LV pressure, volume, and the electrocardiogram were displayed and digitized by using an off-line computer. LV pressurevolume relationships were determined by using transient compressions of the inferior vena cava. The data were recorded as a series of pressurevolume loops (approximately 20), which were analyzed by using Integral 3 software (Unique Medical Co.) [14].

We then determined the indices for systolic and diastolic LV functions. The maximal and minimal rates of change in LV pressure $(\mathrm{dP} / \mathrm{dt}$ max and $\mathrm{dP} / \mathrm{dt}$ min, respectively) were obtained from steadystate beats by using custom-made software. We also assessed the early and active part of relaxation by using the relaxation time constant $(\tau)$, which was determined by fitting with the LV pressure decay. We also measured LV end-systolic and end-diastolic pressures (LVESP and LVEDP, respectively).

LV function was assessed by systolic and diastolic pressure-volume relationships, which were derived from the pressure-volume loops (ESPVR and EDPVR, respectively). Those parameters were obtained as linear fits to the LVESP and LVEDP pressure-volume points, respectively, and characterized by the slope.

\section{Angiography}

Eight weeks after the treatment, gastroepiploic angiography was performed to determine newly developed collateral vessel communications between the omentum and myocardium. Following the induction of anesthesia, a catheter was introduced into the left GEA through the right femoral artery. Contrast medium $(5 \mathrm{~mL})$ was manually 
Citation: Shudo Y, Miyagawa S, Fukushima S, Kainuma S, Saito A, et al. (2014) Promising Therapeutic Effects of Cell Sheet Implantation with a Pedicle Omentum Flap to Enhance the Angiogenic Response to Ischemic Cardiomyopathy. J Stem Cell Res Ther 4: 159. doi:10.4172/21577633.1000159

Page 3 of 7

injected through the catheter into the left GEA at a continuous rate for 10 seconds. Several angiograms of the arteriovenous phase were taken in each animal.

\section{$\mathrm{X}$-ray micro-CT analysis}

The mini-pigs in the Cell sheet+Omentum group were prepared for microvascular visualization. The entire heart with the omentum was resected and fixed with $4 \%$ paraformaldehyde solution overnight $\left(4^{\circ} \mathrm{C}\right)$. X-ray micro-CT was performed to visualize the microvascular network in vivo [15-17]. The contrast medium was prepared as previously described [18] and consisted of 95\% Iomeron 350 (Eisai, Tokyo, Japan) and 5\% gelatin (Sigma-Aldrich, St. Louis, MO, USA). Polyethylene tubing was inserted into the GEA, and warmed contrast medium $\left(60^{\circ} \mathrm{C}\right)$ was injected in a continuous infusion (at $3 \mathrm{~mL} / \mathrm{min}$ ) until the contrast medium enabled filling up to the microvascular level with no or minimal filling of the capillaries. The main purpose of this image was to establish the presence of collateral vessels between the implanted myocardium and the omentum.

\section{Cell tracking system by magnetic resonance imaging}

SMB cells were magnetically labeled before treatment by using a super paramagnetic iron oxide (SPIO) with a hemagglutinating virus of Japan (GenomONENeo; Ishihara Sangyo Kaisha Ltd., Osaka, Japan). The SPIO-labeled areas were measured and corrected by the SPIO density values of interest at the mid-LV level (clearly depicting the base of the papillary muscles) [19]. MRI was performed on a 1.5T MR scanner (GE Medical Systems, Milwaukee, WI, USA) by using electrocardiography gating before and 8 weeks after the treatment.

\section{Histological analysis}

To detect the fate of SMB, cryosections were stained with an antidesmin antibody (1:200 dilution; Dako, Glostrup, Denmark) and anti-vimentin antibody (1:200 dilution; Dako). At 8 weeks after the treatment, phthalocyanine blue (Wako, Osaka, Japan) was used to examine blood perfusion in the engineered myocardial tissues. Three hundred micro-liters of phthalocyanine blue was injected into the LAD just distal to the site where the ameroid constrictor was placed. The sections were also stained with an antibody to CD68 (1:100 dilution; Dako) to assess the inflammatory effect within 5 randomly selected fields in the border area. The sections were also stained with an antibody to proliferating cell nuclear antigen (PCNA) (1:200 dilution; Dako) to assess the cellular proliferative activity in 5 randomly selected fields in the border area.

\section{Statistical analysis}

Continuous values are expressed as the mean \pm SD. Comparisons between pre- and post-treatment values were made by using the unpaired $t$ test. For comparisons among 4 groups, we used 1-way analysis of variance, followed by Fisher's protected least-significance difference test. A p value $<0.05$ was considered statistically significant. All statistical calculations were performed by using SPSS software (version 11.0; SPSS Inc., Chicago, IL, USA).

\section{Animal care and biosafety}

The mini-pigs were obtained from Japan Farm Co. Ltd., Kagoshima, Japan. Food and water were provided ad libitum. All studies were performed with the approval of the institutional ethics committee of Osaka University. Humane animal care was used in compliance with the "Principles of Laboratory Animal Care" formulated by the National Society for Medical Research and the "Guide for the Care and Use of Laboratory Animals" prepared by the Institute of Animal Resources and published by the National Institutes of Health (Publication No 8523, revised 1996).

\section{Results}

\section{Implantation of skeletal myoblast cell sheets with omentum increased cardiac function}

The effects of SMB cell sheet with omentum implantation on cardiac function were assessed in a mini-pig ICM model. Hemodynamic studies showed that ESPVR were significantly improved in the Cell sheet+Omentum group compared to the other groups. The Cell sheet+Omentum group also showed significantly better improvement of $\mathrm{dP} / \mathrm{dt}(\max )$ and LVEDP compared to the Omentum and ICM groups. There was no significant difference in the active part of relaxation shown by the relaxation time constant $(\tau)$ among these groups (Table 1).

\section{Implantation of skeletal myoblast cell sheets with omentum increased myocardial blood perfusion}

Myocardial blood flow was quantitatively assessed by performing MCE before and 8 weeks after the treatment. Myocardial blood flow was similar among all 4 groups prior to treatment. Blood flow significantly increased after cell sheet implantation, but the blood flow in the Cell sheet+Omentum group was significantly larger than that in the Cell sheet group (Figure 2).

\section{In vivo micro-vascular collateral vascular formation within the tissue-engineered myocardium}

Eight weeks after treatment, angiography via the GEA showed rich collateral vascular-rich collateral formation that potentially perfused the omental flap and the myocardial capillary beds in the mini-pig of

\begin{tabular}{|c|c|c|c|c|c|c|c|c|}
\hline & \multicolumn{2}{|c|}{ Cell-sheet with omentum group } & \multicolumn{2}{|c|}{ Cell-sheet group } & \multicolumn{2}{|c|}{ Omentum group } & \multicolumn{2}{|c|}{ ICM group } \\
\hline & Baseline & 8 weeks & Baseline & 8 weeks & Baseline & 8 weeks & Baseline & 8 weeks \\
\hline \multicolumn{9}{|c|}{ Pressure volume analysis } \\
\hline ESPVR $(\mathrm{mmHg} / \mathrm{s})$ & - & $18 \pm 1^{\dagger, \ddagger}{ }^{*}$ & - & $11 \pm 2$ & - & $10 \pm 4$ & - & $8 \pm 2$ \\
\hline $\mathrm{dP} / \mathrm{dt}(\max )(\mathrm{mmHg} / \mathrm{s})$ & - & $1600 \pm 130 \ddagger$ & - & $1300 \pm 120$ & - & $1100 \pm 130$ & - & $940 \pm 100$ \\
\hline $\mathrm{dP} / \mathrm{dt}(\mathrm{min})(\mathrm{mmHg} / \mathrm{s})$ & - & $-1600 \pm 300^{*}$ & - & $-1500 \pm 260$ & - & $-1200 \pm 260$ & - & $-1100 \pm 120$ \\
\hline Tau (msec) & - & $59 \pm 18$ & - & $56 \pm 13$ & - & $54 \pm 8$ & - & $50 \pm 10$ \\
\hline LVESP (mmHg) & - & $81 \pm 14$ & - & $75 \pm 16$ & - & $74 \pm 13$ & - & $78 \pm 26$ \\
\hline LVEDP (mmHg) & - & $6 \pm 2^{\ddagger,^{*}}$ & - & $7 \pm 1$ & - & $10 \pm 2$ & - & $12 \pm 3$ \\
\hline
\end{tabular}

ICM: Indicates Ischemic Cardiomyopathy; ESPVR: End-Systolic Pressure Volume Relationship; LVESP: Left Ventricular End-Systolic Pressure; LVEDP: Left Ventricular End-Diastolic Pressure; LV: Left Ventricular. Date are presented as mean \pm SE. ${ }^{\dagger} p<0.05$ vs. Cell-sheet group, ${ }^{\ddagger} p<0.05$ vs. Omentum group, ${ }^{*}<<0.05$ vs. ICM group

Table 1: Implantation of skeletal myoblast cell sheets with omentum increased cardiac function, as shown by hemodynamic study. 
Citation: Shudo Y, Miyagawa S, Fukushima S, Kainuma S, Saito A, et al. (2014) Promising Therapeutic Effects of Cell Sheet Implantation with a Pedicle Omentum Flap to Enhance the Angiogenic Response to Ischemic Cardiomyopathy. J Stem Cell Res Ther 4: 159. doi:10.4172/21577633.1000159

Page 4 of 7

the Cell sheet+Omentum group (Figure 3A). Moreover, X-ray micro$\mathrm{CT}$ detected the presence of in vivo newly developed microvascular collateral vessels in the implanted myocardium (Figure 3B).

\section{Implantation of skeletal myoblast cell sheets wrapped with omentum improved cell retention}

Cell retention was quantitatively assessed by performing cardiac MRI and the ironoxide-labeling method to compare the efficacy between the Cell sheet+Omentum and Cellsheet groups (Figure 4A). Cell retention was similar between the 2 groups prior to treatment. At 8 weeks after treatment, there was significantly greater cell retention of the implanted SMB in the Cell sheet+Omentum group compared with in the Cell sheet group, indicating the beneficial effects of omentum on the SMB cell sheets (Figure 4B).

Left ventricular remodeling and fate of skeletal myoblast following implantation of skeletal myoblast cell sheets with omentum

The short axis-area of the LV was small compared with that of the ICM as assessed by macroscopic images (Figure 5A). Desmin-positive and vimentin-negative cells were identified in the implanted cell sheets following SMB cell sheet with omentum implantation (Figure 5B).

Skeletal myoblast cell sheets with omentum attenuated inflammation and enhanced cell proliferation activity

Implantation of SMB cell sheets with omentum significantly attenuated the inflammatory effect in the border area compared to that

(A)

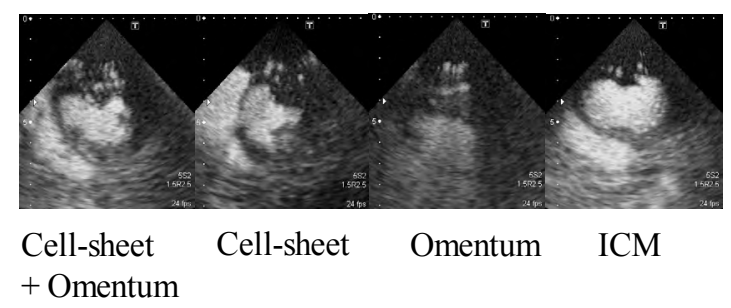

(B)

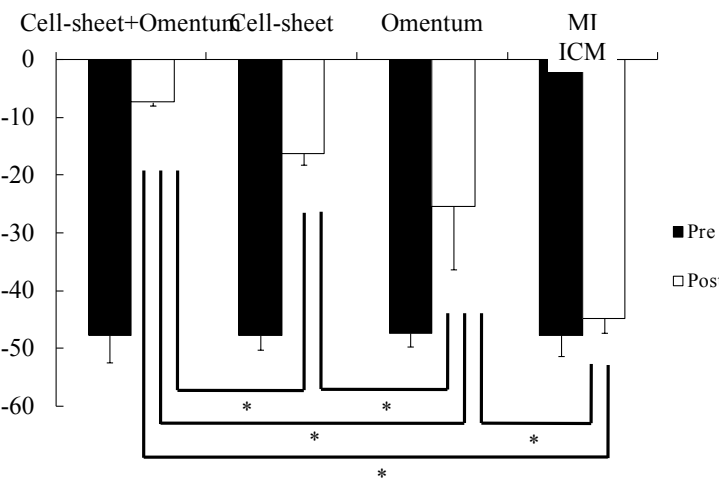

Pre: preoperative; Post: postoperative. " $p<0.05$

Figure 2: Blood flow analysis by performing myocardial contrast echocardiography. (A) Representative left ventricular short-axis view of 1.5 harmonic imaging at 8 weeks after the treatment. (B) Quantification of blood flow. Postoperatively, blood flow was significantly increased in the Cell sheet+Omentum, Cell sheet, and Omentum groups. Postoperatively, blood flow was the highest after cell sheet with omentum implantation.
(A)

(B)
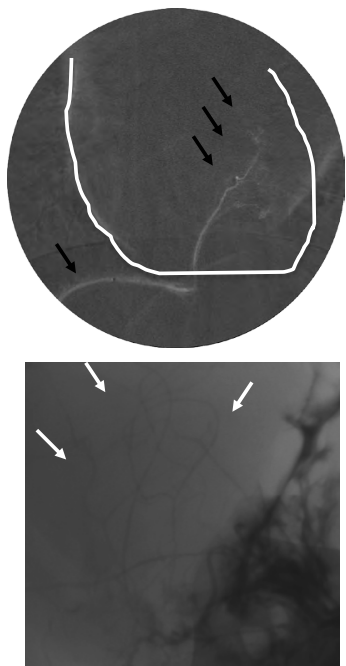

Figure 3: Collateral vessel analysis via the gastroepiploic artery. (A) Gastroepiploic artery (black arrow) perfused the omentum and the myocardial tissue as detected by angiography via the gastroepiploic artery. The white broken line indicates the edge of the heart. (B) Representative image of the presence of microvascular collateral vessels (white arrow) in the treated myocardium by $\mathrm{X}$-ray micro-computed tomography.

(A) Cell-sheet + Omentum group
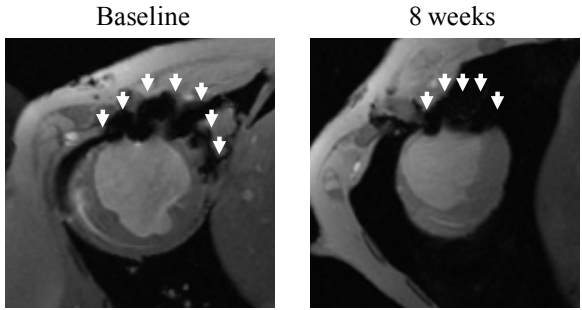

Cell-sheet group Baseline

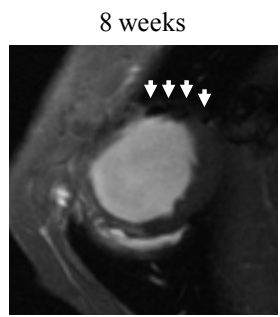

(B) SPIO labeling ratio (\%)

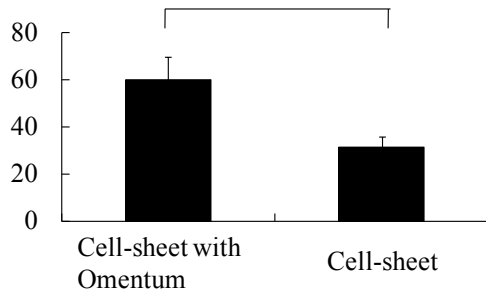

MRI, magnetic resonance imaging; SMB, skeletal myoblast. White arrow shows the low intensity of the super paramagnetic iron oxide-labeled area

Figure 4: Cell tracking system by performing MRI. (A) MRI was examined just prior (baseline) and 8 weeks after SMB cell sheet implantation with or without omentum. (B) Cell retention was quantitatively assessed by performing MRI and the ironoxide-labeling method at 8 weeks corrected by that at day 0 . There was significantly more cell retention of implanted SMB in the Cell sheet+Omentum group compared with in the Cell sheet group. 
Citation: Shudo Y, Miyagawa S, Fukushima S, Kainuma S, Saito A, et al. (2014) Promising Therapeutic Effects of Cell Sheet Implantation with a Pedicle Omentum Flap to Enhance the Angiogenic Response to Ischemic Cardiomyopathy. J Stem Cell Res Ther 4: 159. doi:10.4172/21577633.1000159

Page 5 of 7

of SMB cell sheets only as demonstrated by anti-CD68 staining (Figures $5 \mathrm{C}$ and 5D). A greater number of PCNA-positive cells were identified in the border myocardium following SMB cell sheet with omentum implantation compared to the other groups (Figures $5 \mathrm{E}$ and $5 \mathrm{~F}$ ).

\section{Formation of collateral vascular network after implantation of SMB cell sheets with omentum}

Phthalocyanine blue (Wako) was infused into the coronary artery and the vascular network was examined in the engineered cell sheet construction. Blood vessels within the cell sheet construct stained blue, indicating an anastomotic connection between the host coronary vasculature and the blood vessels within the implanted cell sheet construct (Figure 6).

\section{Discussion}

This study revealed a multifaceted mechanism by which the targeted implantation of SMB cell sheets with omentum enhances myocardial function in a porcine model of ICM. Significant angiogenic and antiinflammatory effects were observed in vivo where SMB cell sheets with omentum stimulated blood vessel formation; this effect is a fundamental component of the augmented blood flow demonstrated in vivo. The observed increased angiogenesis and improved blood perfusion in the implanted site reflected the significant in vivo angiogenic potential of this technology, which accelerated the growth of therapeutic vessels into the host ischemic myocardium. More importantly, the cell sheets and omentum implanted into ischemic hearts that formed anastomotic communications with the non-perfused native vasculature in the damaged myocardiummay efficiently supply blood to ischemic myocardium through newly formed vasculature between the omental and recipient coronary vessels.

With regard to cell engraftment, the data clearly demonstrated prolonged cell retention of the implanted SMB cell sheets onto the myocardium through improved blood perfusion in the implanted cell sheet. Inflammation in the implanted site was reduced, which was induced by omental implantation. SMB cell sheet with omentum implantation had a robust angiogenic effect that translated to enhanced myocardial function of the ischemic heart. Moreover, this combination therapy may be applicable in clinical situations, and additional omentopexy may enhance the therapeutic efficacy of myoblast sheet implantation in patients with ICM.

The omentum flap has often been employed by cardiothoracic surgeons due to its high potential to stimulate revascularization and provide nutrients and oxygen $[20,21]$.In the present study, the omentum was used to provide blood supply to the ischemic heart in patients with multiple diffuse coronary artery disease. It has also been reported to be able to induce capillary vessels, large vessels, and possibly mature new blood vessels. However, the development of mature newly formed vessels from the coronary artery or myocardium was historically limited, and the blood supply was insufficient in clinical settings [20].
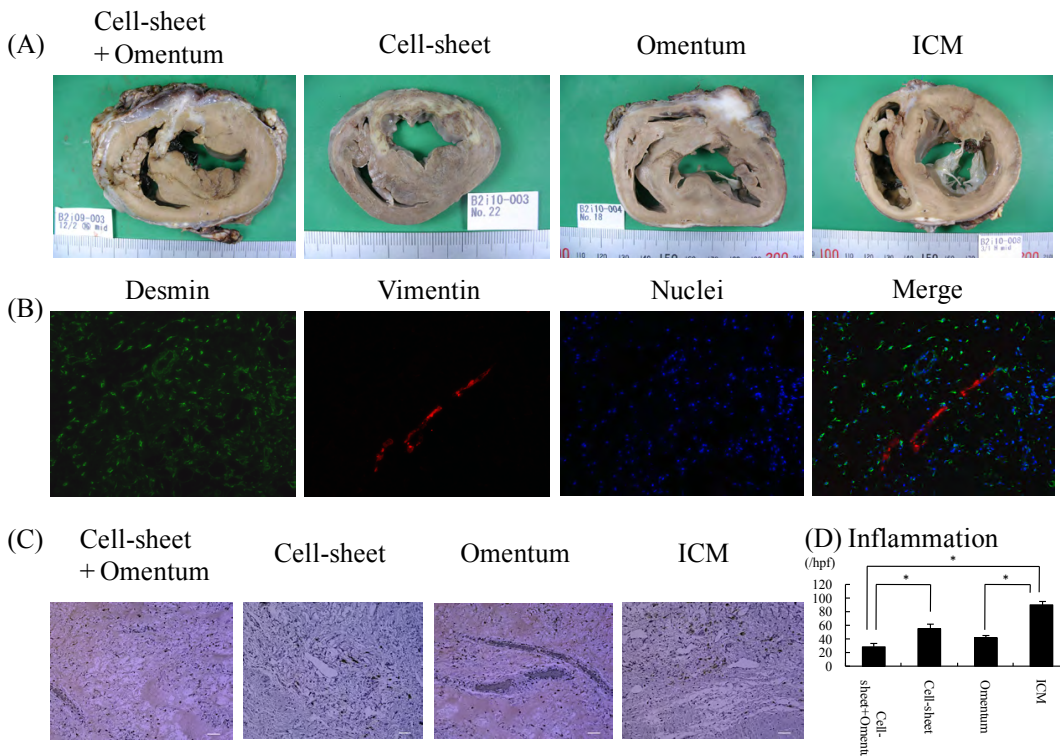

(E) Cell-sheet
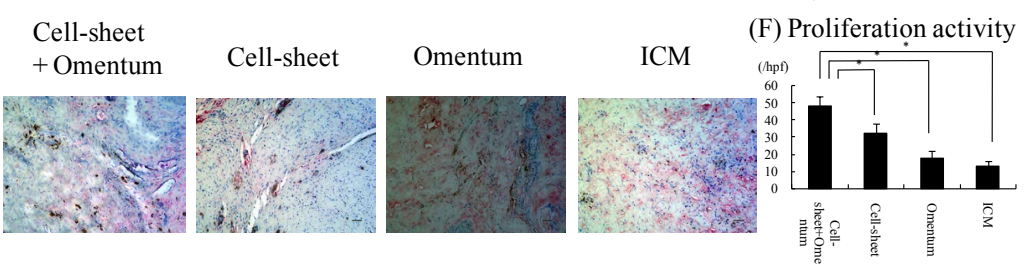

Figure 5: Histological findings of extirpated hearts after treatment. (A) Macroscopic images of impaired myocardium after treatment. (B) Immunostaining fordesmin and vimentin showing desmin-positive andvimentin-negative cells in the implanted cell sheets following SMB cell sheet with omentum implantation. Green, desmin; red, vimentin; blue, nuclei. (C) Representative anti-CD68 staining of the border myocardium. (D) Quantification of inflammation. Inflammation at the border was significantly suppressed in the SMB Cell sheet+Omentum group compared with the SMB Cell sheet only group. (E) Representative anti-PCNA staining of the border myocardium. (F) Quantification of cell proliferative activity. Proliferative activity was significantly enhanced in the Cell sheet with Omentum group compared with the other groups. " $\mathrm{p}<0.05$. SMB: skeletal myoblast; PCNA: proliferative cell nuclear antigen. 
Citation: Shudo Y, Miyagawa S, Fukushima S, Kainuma S, Saito A, et al. (2014) Promising Therapeutic Effects of Cell Sheet Implantation with a Pedicle Omentum Flap to Enhance the Angiogenic Response to Ischemic Cardiomyopathy. J Stem Cell Res Ther 4: 159. doi:10.4172/21577633.1000159

Page 6 of 7

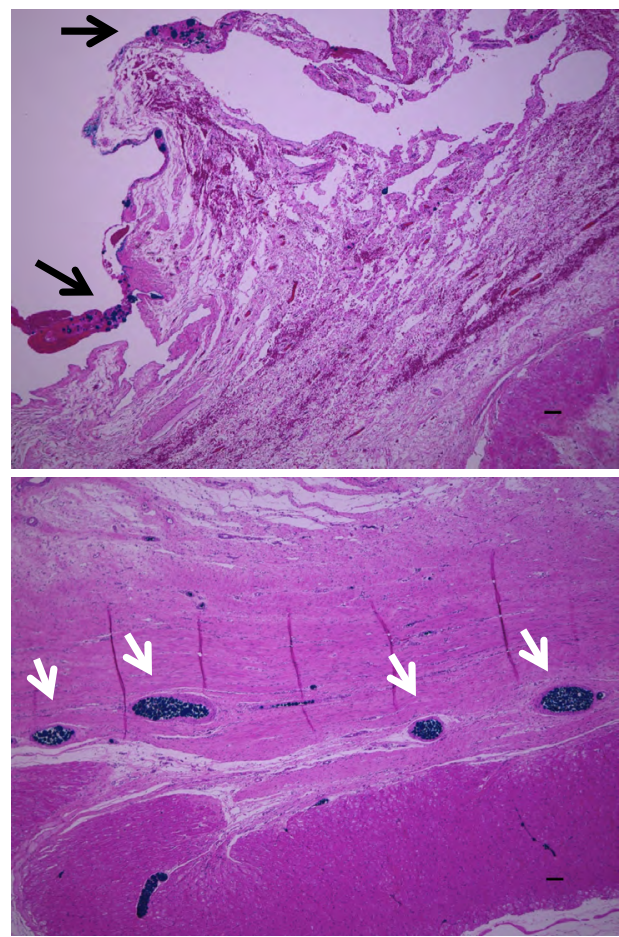

Figure 6: Phthalocyanine blue was infused into the coronary artery and vascular formation was examined in the engineered myocardial tissues. Blood vessels within the cell sheet construct stained blue within the host coronary vasculature (white arrow) and the implanted cell sheet construct (black arrow)

Although our study also proved that omentum implantation induced angiogenesis in a porcine infarction model, the degree of angiogenesis is insufficient to improve cardiac performance. As such, an adjuvant armamentarium may be crucial to achieving significantly improved cardiac function when the omentum implantation technique is used. What would comprise a suitable adjuvant armamentarium for enhancing omentum implantation-induced angiogenesis? Capillary formation has been reported to occur via two basic vessel-constructing processes: angiogenesis, i.e., the formation of new capillaries via sprouting or intussusception from pre-existing vessels, and vasculogenesis, which is the de novo formation of vasculature that occurs in the developing embryo [22]. Angiogenesis has also been reported to require a dynamic temporal and spatially regulated interaction between endothelial cells, pericytes, extracellular matrices (ECM), and angiogenic factors [23]. Angiogenic cytokines, in particular, may be important factors for enhancing omentum implantation-induced angiogenesis. Although it has already been reported that the implantation of $\beta$-fibroblast growth factor gelatin sheets and omentum significantly impacted angiogenesis in a rat infarction model, this method may only induce a paracrine effect of cytokines and may not be able to provide an extracellular matrix unlike fibronectin, which can provoke effective angiogenesis [21]. As such, supplying cytokines only may not be the best way to induce effective angiogenesis.

On the other hand, our group investigated the effects of SMB as a neovasculogenic therapy for ICM by using an SMB cell intramyocardial injection [24], a tissue-engineered cell sheet technique [25,26], and a multi-layered cell sheet technique [9]. We employed cell sheet technology, which provides efficient delivery of cells onto the ischemic area of the myocardium with minimal myocardial injury and cell dispersion and preserves cell-cell and cell-ECM architectural structure $[6,8,9]$. The special feature of the cell sheet may be that it produces several kinds of cytokines such as hepatocyte growth factor (HGF) or vascular endothelial growth factor (VEGF) and, most importantly, may provide several extracellular matrixes such as fibronectin to recipient myocardium and direct the growth of existing vessels of the omentum to the damaged myocardium (data not shown).Moreover, the cytokine cocktail secreted from the cell sheet may be superior to any single cytokine for angiogenesis [27].

It has also been reported that the secretion of VEGF and transforming growth factor- $\beta$ is thought to promote stabilization in the synthesis and deposition of the extracellular matrix as well as the contextual regulation of proliferation and differentiation [28]. In this combined therapy, cytokines and extracellular matrix secreted from the myoblast sheet may play a crucial role in directing the growth of existing omentum vessels to the recipient myocardium, and the growing vessels functionally connect to the native coronary artery, resulting in the so-called vasculogenesis.Our present study, in which a well-organized capillary network between the omentum and the recipient myocardium was demonstrated by $\mathrm{X}$-ray micro-CT analysis, may prove that hypothesis.

Cell engraftment is another critical aspect of myocardial regeneration. The potential advantages of cell sheet technology have been reported to include the delivery of a larger number of transplanted cells and the integration with native tissues without destroying the cell-cell or cell-ECM adhesions in the cell sheet $[9,10]$. Based on these findings along with our significant findings of increased cell retention and the enhanced secretion of HGF in vitro from the SMB cell sheet, we believe that it is likely that the SMB cell sheet, with the aid of the omentum, increased cell retention by preventing apoptosis, which could be mediated by ECM receptors or modulated by growth factors (e.g. HGF) [29]. An anti-inflammatory effect is one more important benefit of combination cell sheet and omentum implantation. It is postulated that anti-inflammatory cytokines (e.g. interleukin-10) secreted from SMB cell sheets suppress the proliferation of macrophages with the aid of the omentum, which was thought to attenuate inflammation. Importantly, the fact that this therapy yielded marked cardioprotective effects through this anti-inflammatory effect should help it prolong cell retention.

In conclusion, we demonstrated here that the implantation of SMB cell sheets with omentum enhanced the angiogenic effect by facilitating more mature microvascular formation and increasing cell retention. We also observed that the SMB cell sheet with omentum technology initiated robust angiogenesis, thereby increasing blood perfusion, enhancing myocardial function, and improving global ventricular function in this porcine ICM model. This concept may lead to new regeneration therapies in advanced cardiomyopathy.

\section{Sources of Funding}

The present study was supported by Grants from the JSPS Core-toCore Programand Health and labor, Science Research Grants, Research on Regenerative Medicine for Clinical Application, Japan.

\section{Acknowledgements}

We wish to thank Mr. Shigeru Matsumi for his excellent technical assistance.

\section{References}

1. Menasche P, Hagege AA, Scorsin M, Pouzet B, Desnos M, et al. (2001) Myoblast transplantation in heart failure. Lancet 357: 279-280. 
Citation: Shudo Y, Miyagawa S, Fukushima S, Kainuma S, Saito A, et al. (2014) Promising Therapeutic Effects of Cell Sheet Implantation with a Pedicle Omentum Flap to Enhance the Angiogenic Response to Ischemic Cardiomyopathy. J Stem Cell Res Ther 4: 159. doi:10.4172/21577633.1000159

2. Ghostine S, Carrion C, Souza LC, Richard P, Bruneval P, et al. (2002) Longterm efficacy of myoblast transplantation on regional structure and function after myocardial infarction. Circulation 106: 1131-I136.

3. Hagege AA, Marolleau JP, Vilqiun JT, Alhéritière A, Peyrard S, et al. (2006) Skeletal myoblast transplantation in ischemic heart failure: Long-term follow-up of the first phase I cohort of patients. Circulation 114: I108-I113.

4. Sawa Y, Miyagawa S, Sakaguchi T, Fujita T, Matsuyama A, et al. (2012) Tissue engineered myoblast sheets improve cardiac function sufficiently to discontinue LVAS in a patient with DCM: report of a case. Surg Today 42: 181-184.

5. Shrager JB, Wain JC, Wright CD, Donahue DM, Vlahakes GJ, et al. (2003) Omentum is highly effective in the management of complex cardiothoracic surgical problems. J Thorac Cardiovasc Surg 125: 526-532.

6. Shudo Y, Miyagawa S, Fukushima S, Saito A, Shimizu T, et al. (2011) Nove regenerative therapy using cell sheet covered with omentum flap delivers a huge number of cells in a porcine myocardial infarction model. $\mathrm{J}$ Thorac Cardiovasc Surg 142: 1188-1196.

7. Shudo Y, Miyagawa S, Fukushima S, Saito A, Kawaguchi N, et al. (2011) Establishing New Porcine Ischemic Cardiomyopathy Model by Trans-catheter Ischemia-Reperfusion of the Entire Left Coronary Artery System for Preclinical Experimental Studies. Transplantation 1592: e34-35.

8. Miyagawa S, Sawa Y, Sakakida S, Taketani S, Kondoh H, et al. (2005) Tissue cardiomyoplasty using bioengineered contractile cardiomyocyte sheets to repair damaged myocardium: their integration with recipient myocardium. Transplantation 80: 1586-1595.

9. Sekiya N, Matsumiya G, Miyagawa S, Saito A, Shimizu T, et al. (2009) Layered Implantation of Myoblast Sheets Attenuates Adverse Cardiac Remodeling of the Infarcted Heart. J Thorac Cardiovasc Surg 138: 985-993.

10. Shimizu T, Sekine H, Yang J, Isoi Y, Yamato M, et al. (2006) Polysurgery of cell sheet grafts overcomes diffusion limits to produce thick, vascularized myocardial tissues. FASEB J 20: 708-710.

11. Shudo Y, Nakatani S, Sakaguchi T, Miyagawa S, Yoshikawa Y, et al. (2012) Left ventricular mechanics following restrictive mitral annuloplasty for functional mitral regurgitation: two-dimensional speckle tracking echocardiographic study. Echocardiography 29: 445-450.

12. Asanuma T, Viggen K, Seward JB, Belohlavek M (2002) Dual spectra ultrasonography: An attenuation-compensating technique for myocardial perfusion analysis. J Ultrasound Med 21: 249-259.

13. Toshida T, Ishikura F, Asanuma T, Iwata A, Miki A, et al. (2005) Efficacy of 1.5 harmonic imaging for intravenous myocardial contrast echocardiography. J Echocardiogr 3: 104-108.

14. Kawamura M, Miyagawa S, Miki K, Saito A, Fukushima S, et al. (2012) Feasibility safety, and therapeutic efficacy of human induced pluripotent stem cell-derived cardiomyocyte sheets in a porcine ischemic cardiomyopathy model. Circulation 126: S29-S37.

15. Toyota E, Fujimori K, Ogasawara Y, Kajita T, Shigeto F, et al. (2002) Dynamic changes in three-dimensional architecture and vascular volume of transmura coronary microvasculature between diastolic- and systolic-arrested rat hearts. Circulation 105: 621-626.

16. Toyota E, Warltier DC, Brock T, Ritman E, Kolz C, et al. (2005) Vascular endothelial growth factor is required for coronary collateral growth in the rat. Circulation 112: 2108-2113.

17. Jorgensen SM, Demirkaya O, Ritman EL (1998) Three-dimensional imaging of vasculature and parenchyma in infarct rodent organs with x-ray micro-CT. Am J Physiol 275: H1103-H1114.

18. Wusten B, Buss DD, Deist H, Schaper W (1977) Dilatory capacity of the coronary circulation and its correlation to the arterial vasculature in the canine left ventricle. Basic Res Cardiol 72: 636-650.

19. Kraitchman DL, Heldman AW, Atalar E, Amado LC, Martin BJ, et al. (2003) In vivo magnetic resonance imaging of mesenchymal stem cells in myocardial infarction. Circulation 107: 2290-2293.

20. O'Shaugnessy $L$ (1937) Surgical treatment of cardiac ischemia. Lancet 232 185-194.

21. Tabata K, Jiang C, Nemoto S, et al. (2006) A combination of omental flap and growth factor therapy induces arteriogenesis and increased myocardia perfusion in chronic myocardial ischemia: evolving concept of biologic coronary artery bypass grafting. J Thorac Cardiovasc Surg 132: 891-899.

22. Risau W (1997) Mechanisms of angiogenesis. Nature 386: 671-674

23. Goumans MJ, Valdimarsdottir G, Itoh S, Rosendahl A, Sideras P, et al. (2002) Balancing the activation state of the endothelium via two distinct TGF-beta type 1 receptors. EMBO J 21: 1743-1753.

24. Fujita T, Sakaguchi T, Miyagawa S, Saito A, Sekiya N, et al. (2011) Clinica impact of combined transplantation of autologous skeletal myoblasts and bone marrow mononuclear cells in patients with severely deteriorated ischemic cardiomyopathy. Surg Today 41: 1029-1036.

25. Memon IA, Sawa Y, Fukushima N, Matsumiya G, Miyagawa S, et al. (2009) Repair of impaired myocardium by means of implantation of engineered autologousmyoblast sheets. J Thorac Cardiovasc Surg 130: 646-653.

26. Shudo Y, Miyagawa S, Nakatani S, Fukushima S, Sakaguchi T, et al. (2013) Myocardial Layer-specific Effect of Myoblast Cell Sheet Implantation Evaluated by Tissue Strain Imaging. Circ J 77: 1063-1072.

27. Shudo Y, Miyagawa S, Ohkura H, Fukushima S, Saito A, et al. (2013) Addition of mesenchymal stem cells enhances the therapeutic effects of skeletal myoblast cell-sheet transplantation in a rat ischemic cardiomyopathy model Tissue Eng Part C Methods.

28. Li M, Nishimura H, Iwakura A, Wecker A, Eaton E, et al. (2005) Endothelia progenitor cells are rapidly recruited to myocardium and mediate protective effect of ischemic preconditioning via "imported" nitric oxide synthase activity. Circulation 111: 1114-1120.

29. Zvibel I, Smets F, Soriano H (2002) Anoikis: roadblock to cell transplantation? Cell Transplant 11: 621-630. 